\title{
Chinese Stock Index Futures Price Fluctuation Analysis and Prediction Based on Complementary Ensemble Empirical Mode Decomposition
}

\author{
Ruoyang Chen ${ }^{1}$ and Bin Pan ${ }^{2}$ \\ ${ }^{1}$ School of Economics \& Trade, Hunan University, Changsha, Hunan 410082, China \\ ${ }^{2}$ Financial Research Institute, Wenzhou University, Wenzhou, Zhejiang 325035, China \\ Correspondence should be addressed to Bin Pan; panbin@wzu.edu.cn
}

Received 8 January 2016; Accepted 24 May 2016

Academic Editor: Xiaodong Lin

Copyright (C) 2016 R. Chen and B. Pan. This is an open access article distributed under the Creative Commons Attribution License, which permits unrestricted use, distribution, and reproduction in any medium, provided the original work is properly cited.

Since the CSI 300 index futures officially began trading on April 15, 2010, analysis and predictions of the price fluctuations of Chinese stock index futures prices have become a popular area of active research. In this paper, the Complementary Ensemble Empirical Mode Decomposition (CEEMD) method is used to decompose the sequences of Chinese stock index futures prices into residue terms, low-frequency terms, and high-frequency terms to reveal the fluctuation characteristics over different time scales of the sequences. Then, the CEEMD method is combined with the Particle Swarm Optimization (PSO) algorithm-based Support Vector Machine (SVM) model to forecast Chinese stock index futures prices. The empirical results show that the residue term determines the long-term trend of stock index futures prices. The low-frequency term, which represents medium-term price fluctuations, is mainly affected by policy regulations under the analysis of the Iterated Cumulative Sums of Squares (ICSS) algorithm, whereas short-term market disequilibrium, which is represented by the high-frequency term, plays an important local role in stock index futures price fluctuations. In addition, in forecasting the daily or even intraday price data of Chinese stock index futures, the combination prediction model is superior to the single SVM model, which implies that the accuracy of predicting Chinese stock index futures prices will be improved by considering fluctuation characteristics in different time scales.

\section{Introduction}

As a powerful financial tool, stock index futures can help curb abnormal stock market fluctuations and effectively avoid investment risk by virtue of the primary functions of price discovery, hedging, and arbitrage; moreover, this tool is widely used in both developed and developing countries. The CSI 300 index futures officially began trading on April 15,2010 , which was a significant milepost in the Chinese capital markets. However, the Chinese stock index futures market remains in an emergent state, lacking mature market management mechanisms; thus, shocks (such as government policies, excessive market speculation) often induce wild fluctuations in stock index futures prices. Simultaneously, these price fluctuations can spread rapidly and be amplified by certain trading mechanisms, including margin trades, twoway trades, and mandatory liquidations, which can easily lead to market risk. Therefore, effectively analyzing and accurately forecasting fluctuations in Chinese stock index futures prices is of great theoretical and practical significance for promoting the healthy development of the capital markets.

Many analyses of stock index futures prices frequently use traditional statistical and econometric techniques. For example, Meneu and Torró [1] studied volatility spillovers between the spot and futures markets in Spain using asymmetric multivariate GARCH structures. Zhong et al. [2] investigated the relationship between Mexican stock index futures and the spot market using a modified EGARCH model and cointegration test. Xiong et al. [3] analyzed the long-term and short-term price discovery function of SGX FTSE/Xinhua China A50 Index Futures and A-Share Market using a cointegration test, error correction model, and the impulse response function. Moreover, using highfrequency CSI 300 stock index and futures data in China, 
Zuo et al. [4] investigated the Granger causality and longrun equilibrium between daily realized variance and realized bipower variation and jump variation. Based on five-minute high-frequency data, Chen and Zhang [5] researched the effects of the CSI 300 index futures trading on the jump risk of the spot market by employing a nonparametric method and the Granger causality test. However, there are some key drawbacks with respect to these traditional models. On one hand, many factors (including economic fundamentals, the effects of shocks from significant economic and financial events, and investors' risk preferences [6]) stir up price fluctuations in stock index futures at different time scales. Peters [7] suggested that traders in the financial market would disperse and reflect various time scales due to differences in investment philosophy, ultimately engaging in transactions in different investment time scales: short-term, mediumterm, and long-term. As a new product in an emerging market, Chinese stock index futures have attracted a variety of investor types, and their different investment strategies also have a great impact on stock index futures price fluctuations in different time scales. However, traditional models conduct their analyses based solely on the entire price series, and they cannot explain the inner driving forces of price fluctuations [8]. On the other hand, stock index futures price series are nonlinear and nonstationary financial time series, while traditional models are based on the assumption of linear data and obey a normal distribution; thus, these models are unable to adequately capture the nonlinear patterns in the price series.

The Empirical Mode Decomposition (EMD) method [9] and the Ensemble EMD method [10] are effective tools to address nonlinear, nonstationary data, and these methods can analyze the fluctuation characteristics of a time series indifferent time scales by decomposing them into Intrinsic Mode Functions (IMFs) with independent information. In particular, the EEMD method is a substantial improvement over the EMD method and overcomes the drawback of mode mixing in the EMD method by adding white noise. Thus, the EEMD method is popular and has been widely used and applied in the financial sector. For example, Zhang et al. [8] used the EEMD method to decompose crude oil prices into a trend, with a slowly varying part and a fluctuating process, and then analyzed the long-term, medium-term, and short-term fluctuation characteristics of crude oil prices. Ruan and Bao [11] applied the EEMD method to decompose housing prices into a trend term, a low-frequency term, and a high-frequency term to reveal the intrinsic characteristics of fluctuations in housing prices. Li and Feng [12] employed the EEMD method to separately decompose investor sentiment and the stock index price series into a short-term fluctuation term, a medium-term significant event term, and a long-term trend term, integrating econometric models to expand their research. However, the EEMD method cannot effectively offset the residue noise from the added white noise. Therefore, Yeh et al. [13] further proposed the Complementary Empirical Mode Decomposition (CEEMD) method. This method, which has been used increasingly in recent years $[14,15]$, provides a fast and accurate method for processing data that not only can address the mode mixing in the EMD method but also can completely offset the added residual white noise in the decomposition process that are generated by the EEMD method. However, this latest data processing method has not yet been applied to the stock index futures market.

In addition, by combining with other prediction models, EMD methods can also improve the predictive precision of a single prediction model. For example, Yu et al. [16] built an EMD-Fuzzy Neural Network- (FNN-) Adaptive Linear Neural Network (ALNN) model to forecast crude oil prices; this model yielded superior predictive results in comparison with either a single FNN model or a single ALNN model. In addition, Yang et al. [17] built an EMD-SVM-SVM model to forecast crude oil prices and also improved the predictive results of a single SVM model. Meanwhile, The Support Vector Machine (SVM) model [18] is now widely applied in the stock and stock index futures markets. Huang et al. [19] used the SVM model, a Back Propagation Neural Network (BPNN), Linear Discriminant Analysis (LDA), and other methods to make predictions regarding the Nikkei 225 Index and found that the SVM model exhibited the highest predictive performance among these prediction models. Ince and Trafalis [20] demonstrated that the SVM model has greater precision in predicting stock prices than the Multilayer Perception (MLP) and Autoregressive Integrated Moving Average (ARIMA) methods. Using five real futures contracts, such as the Standard \& Poor 500 stock index futures, Tay and Cao [21] examined the feasibility of SVM modeling by comparing it with a BP neural network; these authors showed that SVMs are better for forecasting futures series. Sai et al. [22] optimized four SVM models with different kernel functions based on the Genetic Algorithm (GA) and the Particle Swarm Optimization (PSO) algorithm; these authors built eight different programs to forecast Chinese stock index futures prices and discovered that the linear kernel function SVM model based on the PSO algorithm yielded superior predictive results for Chinese stock index futures prices. The above papers on the SVM prediction model bolster the conclusion that the SVM model has been used in the stock index futures market, although mainly as a single SVM model.

To improve the analysis of Chinese stock index futures prices and the ability to forecast them, this paper first drew ideas from Zhang et al. [8] and other scholars. Then, we used the CEEMD method to decompose the Chinese stock index futures price series into a residue term, a low-frequency term, and a high-frequency term. Finally, we analyzed the longterm, medium-term, and short-term intrinsic fluctuation characteristics of Chinese stock index futures prices. In addition, substantial fluctuations in the low-frequency term are related to the impact of significant events. To define the events that play a role in the low-frequency term, we use Iterated Cumulative Sums of Squares (ICSS) [23-25] to detect the breakpoints in the low-frequency term. Then, we focus on the problem of forecasting Chinese stock index futures prices by referring to $\mathrm{Yu}$ et al. [16] and Yang et al. [17] to construct a combination prediction model and to forecast Chinese stock index futures prices by building a PSO-based [26-28] CEEMD and SVM combination prediction model. In addition, although previous studies on stock and stock index 
futures markets are based on low-frequency daily, weekly, and monthly price data $[1-3,19-22,29]$, current research regarding intraday high-frequency data gradually became a focus $[4,5,30]$. Therefore, we also use this model to forecast the daily data and the intraday high-frequency data of Chinese stock index futures prices. The main innovation of this paper is the use of the CEEMD method to reveal the intrinsic fluctuation characteristics of Chinese stock index futures prices from various time scales, which provides a new research perspective on the fluctuation analysis of stock index futures prices. The fluctuation characteristics of various scales are introduced into predicting stock index futures prices, thus improving the predictive ability of a single SVM model with respect to the stock index futures market.

The remainder of this paper is structured as follows. Section 2 introduces the methods used in this paper. Section 3 analyzes the Chinese stock index futures prices at various time scales. Section 4 forecasts Chinese stock index futures price. Finally, Section 5 provides a summary.

\section{Introduction of Methodology}

2.1. The CEEMD Method. EMD methods, such as the EMD method, the EEMD method, and the CEEMD method, are self-adaptive data processing methods that can decompose a time series into a series of Intrinsic Mode Functions (IMFs) with independent information. These IMFs are effective tools to address nonlinear, nonstationary data. In this paper, we employ the latest extension, that is, the CEEMD method, to analyze Chinese stock index futures prices. The detailed introduction of the CEEMD method is stated as follows.

The EMD method proposed by Huang et al. [9] is the first method to extract series of IMFs and residue terms from the original time series. In particular, IMFs must satisfy the following two conditions: (1) the number of extrema and zero-crossing points must be equal or differ by a maximum of one and (2) the mean value of the upper and lower envelopes must be zero at all times. Accordingly, decomposition of the EMD method ends when the last residue term (abbreviated as res) $r(t)$ is a monotonic function or a constant; then the original series $x(t)$ can be expressed as

$$
x(t)=\sum_{i=1}^{n} x_{i}(t)+r(t)
$$

where $\mathrm{IMF}_{i}$ is recorded as $x_{i}(t)$. Because mode mixing can easily emerge in the EMD method, Wu and Huang [10] further proposed the EEMD method to address this drawback of the EMD method. The EEMD method assumes that the observed data are amalgamations of true time series and noise and that the ensemble means of data with different noises are closer to true time series. Hence, adding white noise as an additional step may help extract the true IMFs in the time series, and the noise can be offset by ensemble averaging. However, residue noise continues to be generated by the added white noise. Therefore, Yeh et al. [13] then proposed the CEEMD method by adding pairs of positive and negative white noise to generate complementary sequences.
Specifically, the complementary sequences are obtained by the following equation:

$$
\left[\begin{array}{l}
x_{i}^{+}(t) \\
x_{i}^{-}(t)
\end{array}\right]=\left[\begin{array}{cc}
1 & 1 \\
1 & -1
\end{array}\right]\left[\begin{array}{l}
x(t) \\
\varepsilon_{i}(t)
\end{array}\right]
$$

In (2), $\varepsilon_{i}$ represents $i$ th added white noise and $x_{i}^{+}(t)$ and $x_{i}^{-}(t)$ represent the sequences after positive and negative white noise is added for $i$ th time to the original $x(t)$ series. We can generate complementary IMFs via the EMD method, and these are expressed as follows:

$$
\begin{aligned}
& x_{j}^{+}(t)=\frac{1}{n} \sum_{i=1}^{n} x_{i j}^{+}(t), \\
& x_{j}^{-}(t)=\frac{1}{n} \sum_{i=1}^{n} x_{i j}^{-}(t) .
\end{aligned}
$$

Then, the final IMFs with no residue noise are extracted by averaging the two complementary IMFs:

$$
\bar{x}_{j}=\frac{\left(x_{j}^{+}+x_{j}^{-}\right)}{2} .
$$

2.2. ICSS Algorithm. The ICSS algorithm proposed by Inclán and Tiao [23] is a relatively mature approach to detecting breakpoints in sequences. The algorithm assumes that, in the stable time series $Y_{i, t}=\mu_{i}+\varepsilon_{i, t}, \mu_{i}$ denotes the mean return for time series $i$, and $\left\{\varepsilon_{i, t}\right\}$ is the residual series with a mean of 0 and a variance of $\sigma_{i, t}^{2}$. Letting $C_{k}=\sum_{i=1}^{k} \varepsilon_{t}^{2}, k=1, \ldots, T$ be the cumulated sum squares of the residual series $\left\{\varepsilon_{t}\right\}$, the statistic $D_{k}$ can be defined as follows:

$$
D_{k}=\left(\frac{C_{k}}{C_{T}}\right)-\left(\frac{k}{T}\right), \quad k=1, \ldots, T, D_{0}=D_{T}=0 .
$$

If breakpoints do not occur in the variance in the iteration process, the value of the $D_{k}$ statistic will vary around 0 . By contrast, if there are one or more breakpoints in a sequence, the value of the statistic $D_{k}$ will deviate significantly upward or downward from the 0 . Let $k^{*}$ be the value for $K$ at $\max _{k}\left|D_{k}\right|$. If $\max _{k} \sqrt{(T / 2)}\left|D_{k}\right|$ exceeds the preset credible interval, $k^{*}$ will be taken as an estimated break point, where $\sqrt{(T / 2)}$ is a normalizing factor.

2.3. SVM Prediction Model. The SVM model employs a kernel-based method in which the basic idea is to construct a hyperplane with low risk in high-dimensional feature space. This method has the advantage of generalization capabilities and good function approximation. Among the various types of SVM models, the $\varepsilon$-SVM model proposed by Vapnik [18] is mainly used to resolve prediction problems. Given a set of training patterns $\left\{\left(x_{1}, y_{1}\right), \ldots,\left(x_{i}, y_{i}\right)\right\}_{i=1}^{n}$, where $x_{i} \in R$ is the input variable and $y_{i} \in R$ is the output variable, the prediction model is learned from these patterns and is used to forecast 
the output variables of unseen input variables. Specifically, the constraints and objective function for the $\varepsilon$-SVM model are

$$
\begin{aligned}
\min _{\omega, b, \tau, \tau^{*}} & \frac{\omega^{T} \omega}{2}+c \sum_{i=1}^{n} \tau_{i}+c \sum_{i=1}^{n} \tau_{i}^{*} \\
\text { s.t: } & \omega^{T} \phi\left(x_{i}\right)+b-y_{i} \leq \varepsilon+\tau_{i} \\
& \omega^{T} \phi\left(x_{i}\right)+b-y_{i} \geq \varepsilon+\tau_{i}^{*} \\
& \tau_{i}, \tau_{i}^{*} \geq 0, \quad i=1,2, \ldots, n .
\end{aligned}
$$

In (6), $\tau_{i}$ and $\tau_{i}^{*}$ are the slack variables that represent the allowable upper limit and lower limit of training errors, respectively, under the constraint of insensitive loss, $\varepsilon$; $c$ is the penalty factor controlling the degree of penalty on samples whose errors exceed $\varepsilon$.

The Lagrange function must be introduced to resolve (6). According to the Duality Theory and the saddle point condition, the dual form of (6) can be obtained as follows:

$$
\begin{array}{ll}
\min _{\partial^{*}, \partial} & \frac{1}{2} \sum_{i=1}^{n} \sum_{j=1}^{n}\left(\partial_{i}^{*}-\partial_{i}\right)\left(\partial_{j}^{*}-\partial_{j}\right) k\left(x_{i}, x_{j}\right) \\
& +\varepsilon \sum_{i=1}^{n}\left(\partial_{i}^{*}+\partial_{i}\right)-\sum_{i=1}^{n} y_{i}\left(\partial_{i}^{*}-\partial_{i}\right) \\
\text { s.t: } \quad \sum_{i=1}^{n}\left(\partial_{i}^{*}-\partial_{i}\right)=0, \quad 0 \leq \partial_{i}^{*}, \partial_{i} \leq c, i=1,2, \ldots, n \\
\quad \omega=\sum_{i=1}^{n}\left(\partial_{i}^{*}-\partial_{i}\right) .
\end{array}
$$

In (7), $\partial_{i}$ is the nonnegative Lagrangian multiplier of $\partial_{i}^{*}$, and $k\left(x_{i}, x_{j}\right)$ represents the kernel function. Any function that satisfies Mercer's condition can be a kernel function. The common kernel functions include the linear kernel function $k\left(x, x_{i}\right)=x^{T} x_{i}$ and the Radial Basis Function (RBF) kernel function $k\left(x, x_{i}\right)=\exp \left(-\gamma\left\|x-x_{i}\right\|^{2}\right)$. Ultimately, the hyperplane for the underlying prediction problem is given by the following:

$$
f(x)=\sum_{i=1}^{n}\left(\partial_{i}^{*}-\partial_{i}\right) k\left(x_{i}, x\right)+b .
$$

2.4. PSO Algorithm. The PSO algorithm proposed by Kennedy [26] is an effective algorithm to address function parameter optimization. Each particle in the algorithm denoting a potential solution in the solution space has three indicators, including speed, position, and fitness value. The fitness value is determined by the fitness function, which can estimate the merit of the particles. The velocity of the particle determines the movement direction and distance, and the velocity's dynamic adjustment follows the movement experience of the particle itself and other particles, thus enabling the optimal selection of an individual in the solution space. The details are as follows.

Suppose that, in a $D$-dimensional solution space, the population comprising $\mathrm{n}$ particles is $X=\left(X_{1}, X_{2}, \ldots, X_{n}\right)$,

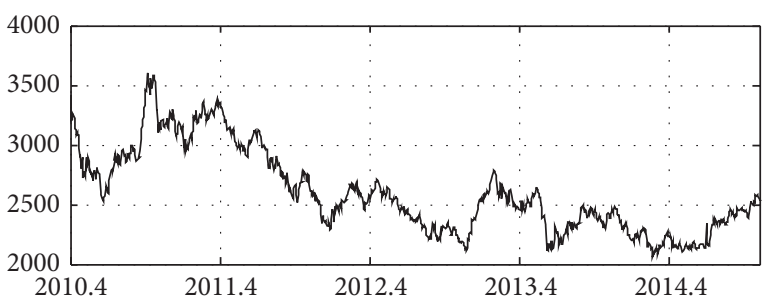

FIGURE 1: Daily closing price of Chinese stock index futures.

the position of $i$ th particle is $X_{i}=\left(x_{i 1}, x_{i 2}, \ldots, x_{i D}\right)^{T}$, and the fitness value of each particle can be calculated based on the objective function and position of each particle. The velocity of $i$ th particle is $V_{i}=\left(V_{i 1}, V_{i 2}, \ldots, V_{i D}\right)^{T}$, its individual extremum is $P_{i}=\left(P_{i 1}, P_{i 2}, \ldots, P_{i D}\right)^{T}$, and the group extremum is $P_{g}=\left(P_{g 1}, P_{g 2}, \ldots, P_{g D}\right)^{T}$. In each iterative process, the particle updates its velocity and position through the individual extremum and the group extremum. The updating equation is as follows:

$$
\begin{aligned}
& V_{i d}^{k+1}=\omega V_{i d}^{k}+c_{1} r_{1}\left(P_{i d}^{k}-X_{i d}^{k}\right)+c_{2} r_{2}\left(P_{g d}^{k}-X_{i d}^{k}\right) \\
& X_{i d}^{k+1}=X_{i d}^{k}+V_{i d}^{k+1}
\end{aligned}
$$

where $\omega$ represents the inertia weight, $d=1,2, \ldots, D, k$ is the current iteration number, $V_{i d}$ is the velocity component of the particle, $c_{1}$ and $c_{2}$ are the acceleration factors and nonnegative constants, and $r_{1}$ and $r_{2}$ are random numbers in the range $[0,1]$. Because the SVM prediction model is sensitive to the model parameters and because the literature [22, 27, 28] indicates that the PSO algorithm performs very well in the parameter optimization process of the SVM model, this paper uses the PSO algorithm to optimize the parameters of the SVM prediction model.

\section{Analysis of Chinese Stock Index Futures Prices in Different Time Scales}

3.1. Data Selection and Description. The CSI 300 index futures are the earliest stock index futures listed in China, and it has been widely used in many studies $[4,5,22]$. Therefore, this paper selects the daily closing price of the stock index futures as this section's research object. The time span runs from April 16,2010 , to November 20, 2014; the sample data total 1,115; and the data are from the Wind database. See Figure 1 and Table 1 for the sample data distribution and the descriptive statistics, respectively.

Nonstationary, nonlinear tests are performed on Chinese stock index futures price series based on Ruan and Bao [11]. Based on the data in Table 1, the null hypothesis that the Chinese stock index future price series are nonstationary cannot be rejected under the condition of less than $10 \%$ significance. Because the postdifferential price series is a stationary series, an autocorrelation analysis is further performed on the series. The test results for $Q$ statistics demonstrate that the null hypothesis for this differential series cannot be rejected under the condition of less than $10 \%$ significance. However, 
TABLE 1: Descriptive statistics for Chinese stock index futures prices.

\begin{tabular}{lcccccc}
\hline & Mean & Std. Dev & JB & ADF & $Q(6)$ & $Q^{2}(6)$ \\
\hline Price series & 2600.691 & 351.503 & $97.269^{* * *}$ & -2.539 & - & - \\
$D$ (price series) & -0.783 & 37.611 & $858.432^{* * *}$ & $-35.531^{* * *}$ & 7.737 & $26.507^{* * *}$ \\
\hline
\end{tabular}

Note. Augmented Dickey-Fuller (ADF) statistics represent the nonstationary test of the price series; $D$ (price series) represents the price series after the first difference; $Q(6)$ statistics represent the autocorrelation test of the time series; $Q^{2}(6)$ statistics represent the autocorrelation test of the square of the time series; and “***" indicates significance at the $1 \%$ significance level.

TABLE 2: Correlations and variance ratios of IMFs and the residue term with the Chinese stock index futures price series.

\begin{tabular}{lccc}
\hline & Mean value & Pearson correlation coefficient & Variance ratio (original series) \\
\hline IMF1 & $0.131(0.238)$ & $0.041(1.363)$ & $0.273 \%$ \\
IMF2 & $-0.096(-0.151)$ & $0.066(2.218)^{* *}$ & $0.372 \%$ \\
IMF3 & $-0.431(-0.523)$ & $0.097(3.273)^{* * *}$ & $0.614 \%$ \\
IMF4 & $-2.103(-1.277)$ & $0.163(5.530)^{* * *}$ & $2.446 \%$ \\
IMF5 & $-4.652(-2.413)^{* *}$ & $0.218(7.454)^{* * *}$ & $3.354 \%$ \\
IMF6 & $46.989(8.007)^{* * *}$ & $0.660(29.329)^{* * *}$ & $31.076 \%$ \\
IMF7 & $-64.873(-18.973)^{* * *}$ & $-0.264(-9.151)^{* * *}$ & $10.550 \%$ \\
Res & $2,625.727$ & $0.762(39.214)^{* * *}$ & $70.278 \%$ \\
\hline
\end{tabular}

Note. The values in parentheses are the $t$ statistics, “**” represents significance at the $5 \%$ level, and “***" represents significance at the $1 \%$ level.

the square of the $Q$ statistics rejects the null hypothesis at less than 1\% significance; in other words, autocorrelation is present. Thus, nonlinear models are more appropriate for studies of Chinese stock index futures prices based on nonstationary, nonlinear tests.

3.2. CEEMD Decomposition. Yeh et al. [13] indicated that the CEEMD method retained the decomposition ability of the EEMD method while effectively offsetting the added white noise. To demonstrate that the advantages of this decomposition remain valid in China's stock index futures prices, this paper uses both the CEEMD and EEMD methods to decompose the China stock index futures price series and then compared the differences between the original series and the sum of the decomposition sequences obtained by the two methods. Depending on the general setting, the standard deviation of the added white noise is 0.2 times the standard deviation of the daily closing price. See Figure 2 for the differences between the results.

As shown in Figure 2, the white noise residue after decomposition by the CEEMD method is nearly zero, whereas a great deal of white noise residue remains after decomposition by the EEMD method, indicating that the CEEMD method can better maintain the completeness of the original series than the EEMD method. Therefore, this paper used the CEEMD method to decompose Chinese stock index futures prices, and the IMFs and residue term are presented in Figure 3.

As shown in Figure 3, the frequency is the highest for IMF1 and then decreased in order among the remaining IMFs. IMF1, IMF2, and IMF3 exhibit dense fluctuations with amplitudes between positive and negative 50 points, approximately. The amplitudes of IMF4 and IMF5 increase to between positive and negative 100 points, and the fluctuation frequency of IMF5 decreases significantly compared with the fluctuation frequency of the previous IMFs, with a fluctuation period of approximately 120 days ( 4 months). The amplitude of IMF6 increases to between positive and negative 200 points-even reaching 400 points once during the initial stock index futures listing period-with a fluctuating period that increases to approximately 400 days (13 months). The upward fluctuation frequency of IMF7 decreases to approximately 100 points, whereas the downward fluctuation frequency is maintained at approximately 200 points; its frequency also decreases to the lowest among the seven IMFs, but the fluctuating period increases to approximately 600 days (20 months). The residue term (res) exhibits a trend of an initial slow decline and subsequent gradual increase, but there is no clear cyclical fluctuation.

As the frequencies decrease, the correlation and variance ratios of the IMFs with the Chinese stock index futures price series increase, indicating that a lower frequency enables greater fluctuation information content to be obtained from the original series. In the low-frequency series, the correlation and variance ratio of IMF6 with the original series is significantly larger than that of IMF5 and IMF7, indicating that medium-term fluctuations in the original series primarily originated from IMF6 and that the correlations and variance ratios of the residue term with the original series are far greater than those of the IMFs, indicating that this series contains the most important information from the original series (Table 2).

3.3. Analysis of Different Time Scales. The analysis described above established that the frequencies of IMF and the residue term differ. The fluctuations of the high-frequency series exhibit ambiguity and disorderliness, and the cyclical fluctuations of the low-frequency series are stronger. The residue term contains most of the information of the original series but does not exhibit a clear fluctuating period, 
TABLE 3: Correlations and variance ratios of the component terms with the Chinese stock index futures price series.

\begin{tabular}{lcc}
\hline & Pearson correlation coefficient & Variance ratio (original series) \\
\hline Residue term & $0.762(39.214)^{* * *}$ & $70.278 \%$ \\
Low-frequency term & $0.526(20.6229)^{* * *}$ & $37.521 \%$ \\
High-frequency term & $0.196(6.661)^{* * *}$ & $4.051 \%$ \\
\hline
\end{tabular}

Note. “***" represents significance at the $1 \%$ significance level.

indicating that the price fluctuation information reflected by the high-frequency series, low-frequency series, and residue term feature more significant differences. To more accurately reveal the fluctuation characteristics of Chinese stock index futures prices at various time scales, we analyze the sum of the IMFs with similar fluctuation characteristics using a $t$ statistics test of the mean from zero [8]. Based on the data in Table 2, the high-frequency IMFs-IMF1, IMF2, IMF3, and IMF4-are summed as the high-frequency term. The lowfrequency IMF-IMF5, IMF6, and IMF7-are summed as the low-frequency term. The residue term alone is retained. The correlations and variance ratios of the three components with the original series are presented in Table 3 , and their distribution is presented in Figure 4.

Consistent with the literature, such as $[8,9]$, the residue term includes most of the information value of the original series and reflects the long-term trend of the original series. In fact, the correlation coefficient of the residue term with the Chinese stock index futures price series in this paper is as high as 0.76 and explains $70 \%$ of the fluctuations in the original price series (see Table 3). Therefore, the residue term can represent the long-term trend of the Chinese stock index futures prices. As shown in Figure 4, between the official listing of stock index futures in China (2010) and 2014, there is a slow downward trend in stock index futures prices. Because the stock index is the "barometer" of a country's macroeconomic situation and because stock index futures serve a price discovery function for stock indexes, the futures market prices reflect newly revealed information more quickly than current market prices. Therefore, macroeconomic changes also determine the long-term trend of stock index futures prices. As China's Gross Domestic Product (GDP) growth rate gradually slows after 2010 (the GDP growth rates from 2010 to 2014 are $10.45 \%, 9.3 \%, 7.65 \%, 7.67 \%$, and $7.4 \%$, resp.) (data for GDP growth rates in China from 2010 to 2014 were downloaded from the National Bureau of Statistics of the People's Republic of China website (http://www.stats.gov.cn/)) and macroeconomic growth shows signs of fatigue, stock index futures prices also decrease year by year, decreasing from 3,376 points in the beginning of 2010 to 2,332 points in the beginning of 2014, with an average annual growth rate of approximately $-11.192 \%$. However, this downward trend slows after 2014 and reverses slightly in the second half of 2014 mainly because in that year, certain important and significant political actions, including administrative simplification and anticorruption legislation and enforcement, create a healthy political environment for the economy. In addition, a reduction in the reserve requirement ratio and an interest rate cut will free up cash for investment, and China's economic growth turns from incremental expansion to an optimized structure, which results in good prospects for investors. Accordingly, more money is flowing into the stock market, which drives stock index prices higher, and higher stock index futures price result simultaneously.

The low-frequency term mainly reflects the mediumterm effect of significant events on stock index futures prices. As shown in Table 3, the correlation between the lowfrequency term and the Chinese stock index futures price series is 0.56 and explains up to $37.5 \%$ of the original price series, indicating that significant events in recent years play an important role in the fluctuations in the Chinese stock index futures prices. Figure 4 illustrates the plummet that occurs during the launch of the Chinese stock index futures, which has a maximum amplitude of 500 points. This plummet is mainly due to the strengthening of the country's efforts to suppress real estate market speculation in 2010. This initiative should lead to a stock market decline because real estate investment is an important economic driver at the time, but the stock market does not make the appropriate response. The Chinese stock index futures unleash their price discovery function after launch, which leads to adjustments in stock index prices. In addition, many investors use the hedging function of stock index futures to avoid the risk of a stock market crash. However, after the substantial fluctuations during launch, the amplitude of the low-frequency term decreases slightly, reflecting in part the stabilization effect of stock index futures on the stock market. To further investigate the effects of significant events on Chinese stock index futures prices, we use the ICSS algorithm to perform breakpoint tests on the rate of change in the low-frequency term (first-difference processing on the low-frequency term to identify the breakpoints). Table 4 presents the results of the breakpoint test on the low-frequency term. The lowfrequency term exhibits 11 breakpoints or, in other words, 12 different volatility mechanisms. The significant events that cause breakpoints are primarily administrative macrocontrol policies, which are mainly addressed by adjusting monetary policy, perfecting the capital market mechanism and promoting market liberalization. The execution of these policies brings volatility to Chinese stock index futures, in turn creating a new volatility mechanism. These breakpoints are mainly concentrated in 2012 and 2013. During these two years, the government frequently issues regulatory policies to bolster financial deepening and reform, and investors adopt a medium-term investment strategy revolving around these policies. As an emerging market, the Chinese stock index futures market is easily affected. However, as the capital market mechanism gradually adjusts, trading in the 


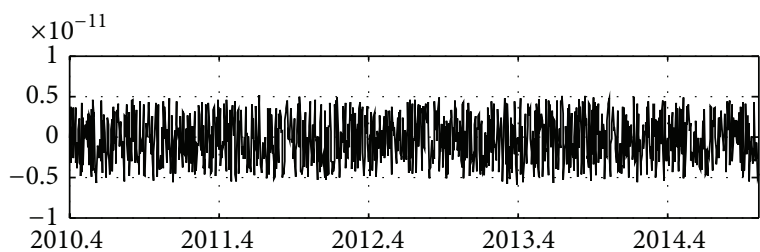

(a)

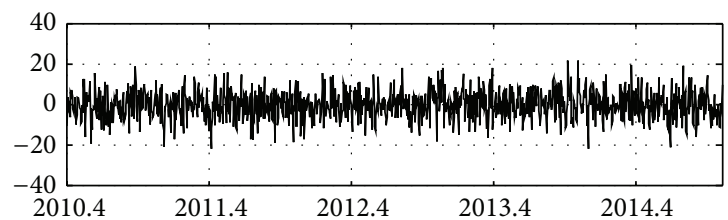

(b)

FIGURE 2: (a) The differences between the stock index futures series and the decomposition sequence obtained by the CEEMD method. (b) The differences between the stock index futures series and the decomposition sequence obtained by the EEMD method.
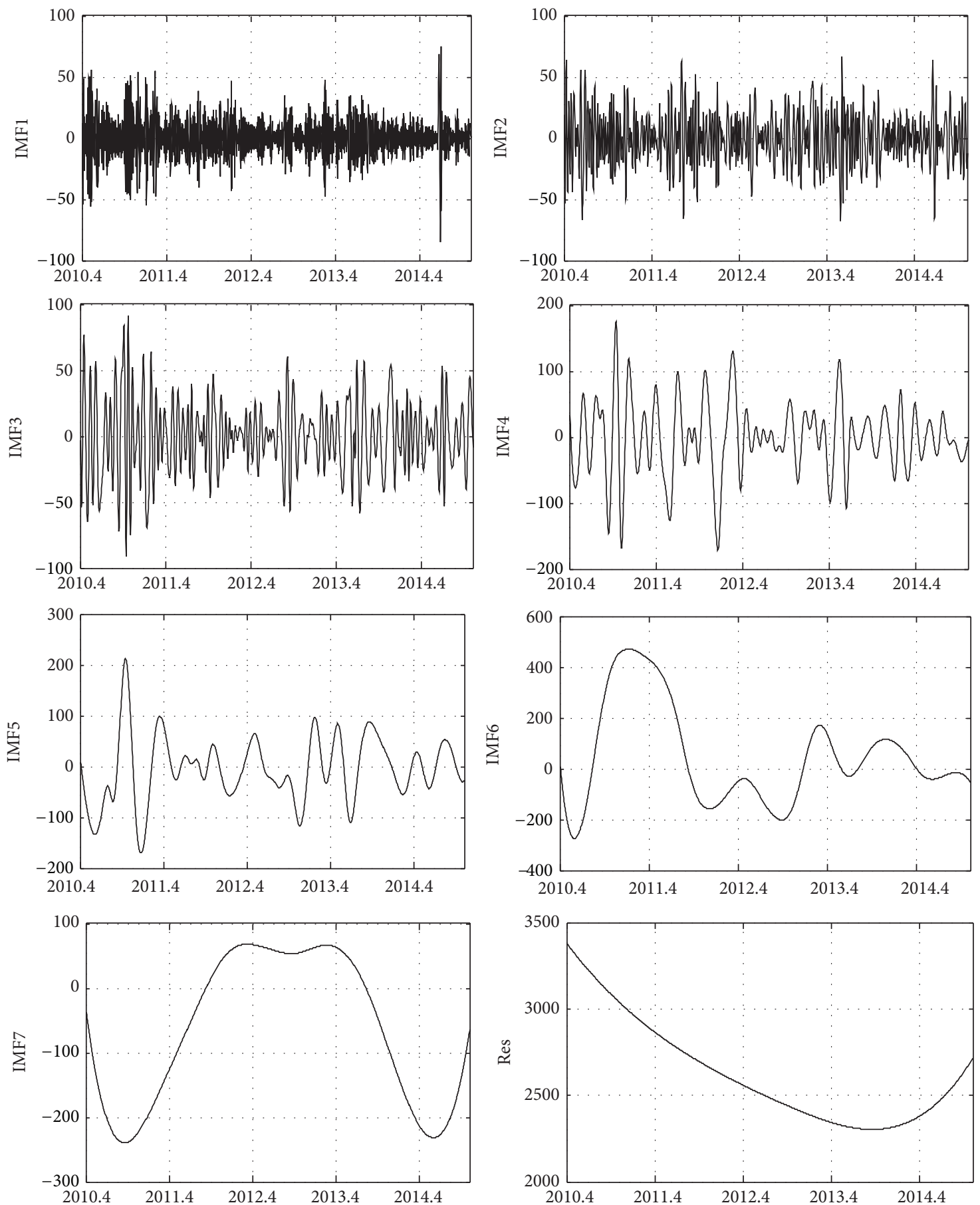

Figure 3: IMFs and residue term. 
TABLE 4: Breakpoint tests on the low-frequency term and corresponding significant events.

\begin{tabular}{llll}
\hline $\begin{array}{l}\text { Breakpoint } \\
\text { number }\end{array}$ & Corresponding time & Corresponding significant event & The response of stock index futures prices \\
\hline 1 & December 13,2010 & $\begin{array}{l}\text { On December 10, 2010, the Central } \\
\text { Economic Working Conference convenes } \\
\text { and indicates that the moderately loose } \\
\text { monetary policy has become robust }\end{array}$ & $\begin{array}{l}\text { This event implies that the supply of } \\
\text { liquidity to the capital market would be } \\
\text { reduced, which was bad news for the stock } \\
\text { index futures market. Hence, the stock } \\
\text { futures prices slump }\end{array}$ \\
\hline \multirow{2}{*}{ September 28,2011 } & $\begin{array}{l}\text { In October 2011, the credit ratings of a dozen } \\
\text { European financial institutions are } \\
\text { downgraded, and the European debt crisis } \\
\text { further escalates }\end{array}$ & $\begin{array}{l}\text { The Chinese financial market was affected } \\
\text { by this event, and in response, the stock } \\
\text { index futures prices maintained the } \\
\text { downward trend }\end{array}$ \\
\hline
\end{tabular}

From January 6 to 7, 2012, the National Conference on Financial Work convenes in Beijing to set the tone of the next series of major financial reform issues

On May of 2012, the stock index futures prices are affected by a combination of events, including the US stock market and international commodities market crashing and the Chinese PMI index does not continue early rising trend

On June 18, 2010, the Shanghai Stock Exchange and the Shenzhen Stock Exchange separately publish programs related to the

$5 \quad$ June 28, 2012 delisting of listed companies and revise the corresponding Stock Market Rules accordingly, further perfecting the initial public offering (IPO) and delisting systems

On August 16, 2012, the New York branch of the Agricultural Bank of China opens, continuing to accelerate the pace of internationalization of the Chinese banking sector

On October 17, 2012, the Chinese State Council meeting made an important

$7 \quad$ October 18,2012 decision that government would continue to implement the proactive fiscal policy and prudent monetary policy

\begin{tabular}{ll}
\hline January 31, 2013 & $\begin{array}{l}\text { At the end of January 2013, broad money } \\
\text { grew } 15.9 \% \text {, and narrow money grew } 15.3 \%\end{array}$ \\
\hline
\end{tabular}

$9 \quad$ May 21, 2013

In May of 2013, the Chinese macroeconomic situation does not perform well, the Federal Reserve gradually winds up the quantitative easing policy, and many banks in China lack sufficient capital at the time

On September 6, 2013, repackaged treasury note futures debut after a gap of 18 years, which is conducive to the establishing market-oriented pricing benchmarks and advances market-oriented reform of the interest rate

September 10, 2013

11

February 25, 2014
On March 1, 2014, the Central Bank releases the upper limit on interest rates on small amounts of foreign currency deposits in the Shanghai Free-Trade Zone, taking another step toward market-oriented reform of the interest rate
This event makes the stock market a positive prospect for investors and then pushes up the stock index futures prices

The series of significant events poses a serious negative impact on investors' expectations. Therefore, the stock index futures prices stop rising and turn to collapse

This event would improve investors' confidence in the stock market. Hence, the downward trend of stock index futures price is slowed

The event promotes the process of RMB internationalization, which is good news for the financial market, and stock index futures prices inch up

The prudent monetary policy implying limited funds in the capital market was bad news for stock index futures market. Hence, the stock index futures prices tumble over

This event prompted a rise in stock index futures prices

The cumulative effects of shocks of these events occurring in May have an enormous negative influence on the stock index futures market. Hence, the stock index futures prices collapse

This event, rebuilding investors' confidence in the capital market, then pushes up the stock index futures prices

The rise of deposit interest rate indicates that market as risk-free rate would increase, which is negative news for the stock index futures market. Hence, the stock index futures prices plunge 


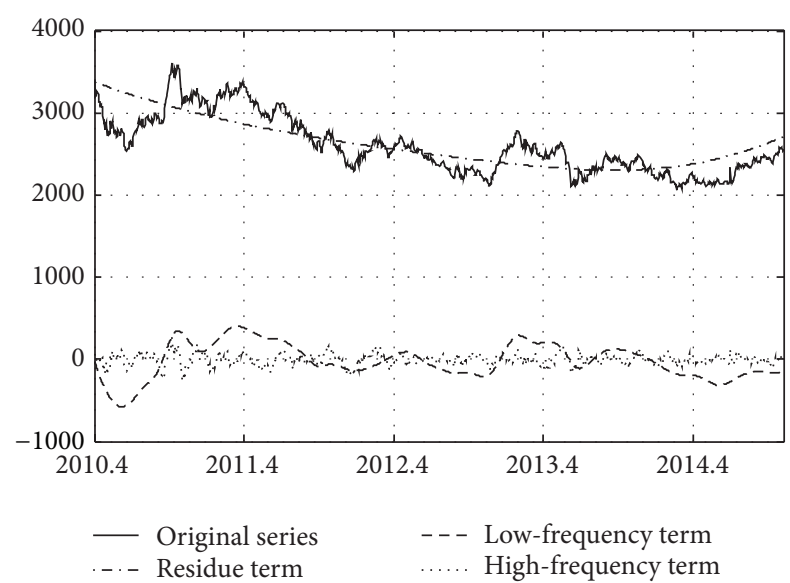

FIGURE 4: Chinese stock index futures prices and the three components.

Chinese stock index futures market further standardizes, and the ability to withstand external impact thus also increases. Therefore, the number of breakpoints decreases after 2014.

The high-frequency term mainly reflects the short-term disequilibrium in the Chinese stock index futures market. As shown in Table 3, the correlation of the high-frequency term with the Chinese stock index futures prices is merely 0.196 , and the ability to explain fluctuations in the original price series is only $4 \%$, indicating that the overall effect of the high-frequency term on Chinese stock index futures prices is limited. However, as Figure 4 shows, the fluctuations of the high-frequency term fit well with the local fluctuations of the Chinese stock index futures prices; in other words, the high-frequency term plays a primary role in local changes in Chinese stock index futures prices. In addition, as opposed to the low-frequency term, the high-frequency term fluctuates frequently but with smaller amplitudes. This difference occurs primarily because the high-frequency term is particularly affected by investors' speculative arbitrage activities. In particular, implementation of a $\mathrm{T}+0$ transaction system in the Chinese stock index futures market means that investors buying and selling stock index futures can complete their transactions in the same day, which suits short-term speculators. Based on this transaction system, there will be more investment chances for investors, which will enhance investors' speculative opportunities. Hence, there are many short-term fluctuations in Chinese stock index futures prices. Meanwhile, it is possible and convenient for investors to access the latest hot investment tip because the role of social interactions is partly substituted for by online information in the Internet age [31]. In reality, this type of new information changes continuously, and investors must frequently adjust their investment strategies to gain more additional benefits by speculating in the spot-futures markets. Therefore, the strong effect of the shock of investors' speculation on Chinese stock index futures prices in the short-term is difficult to sustain. Although fluctuations in the high-frequency term are not important to forecast the long-term trends of Chinese stock index futures, their predictive effect of minor fluctuations on stock index futures cannot be ignored.

\section{Predictions for Chinese Stock Index Futures Prices}

In this section, this paper further investigates the predictions of the Chinese stock index futures prices. In addition to the previous day's price data, we also select the five-minute high-frequency price data of Chinese stock index futures from April 16, 2010, to November 20, 2014, as a research subject, which yields a total of 61,325 data points (the data are obtained from the Wind database). To forecast the daily price, the first 745 (approximately two-thirds) pieces of data are used as the training data, and the last 370 (approximately onethird) pieces of data are used as the test data. To forecast the five-minute high-frequency price, the first 40,883 (approximately two-thirds) data points are used as the training data, and the last 20,442 (approximately one-third) data points are used as the test data.

As our analysis of the price fluctuations of the Chinese stock index futures uses the CEEMD method, the fluctuation characteristics in different time scales have their unique economic implications, we suggest that there may be better predictions of Chinese stock index futures prices when these fluctuation characteristics are considered in the prediction model. Hence, based on the literature [16, 17], a combination prediction model, that is, the CEEMD-SVM-SVM model, is proposed, and the PSO algorithm is used to optimize the parameters of the SVM model. In our empirical research, we perform a one-step-ahead prediction of Chinese stock index futures prices. The specific steps are as follows: (1) the CEEMD method is used to decompose the Chinese stock index futures price series into several IMFs and a residue term; (2) the IMFs are then composed into a low-frequency term and a high-frequency term based on a fine-to-coarse reconstruction, and the residue term remains the same; (3) SVM models are established separately for each of the three components obtained in step (2), and the PSO algorithm is used to optimize the parameters; and (4) the predictive value of the three components obtained in step (3) is used as the input with the real value at the same moment as the output, and then, a SVM combination model is built to obtain the final predictive value. The overall process of the CEEMDSVM-SVM model is shown in Figure 5.

The prediction evaluation indexes are the root mean square error (RMSE), mean absolute prediction error (MAPE), and directional symmetry (DS); the DS prediction evaluation index is used to indicate the model's ability to predict the directionality trend of Chinese stock index futures prices. The prediction evaluation indexes are expressed as follows:

$$
\begin{aligned}
\mathrm{RMSE} & =\sqrt{\frac{1}{n} \sum_{t=1}^{n}[x(t)-\widehat{x}(t)]^{2},} \\
\mathrm{MAPE} & =\frac{1}{n} \sum_{t=1}^{n}\left|\frac{x(t)-\widehat{x}(t)}{x(t)}\right|, \\
\mathrm{DS} & =\frac{1}{n} \sum_{i=1}^{n} d_{i},
\end{aligned}
$$




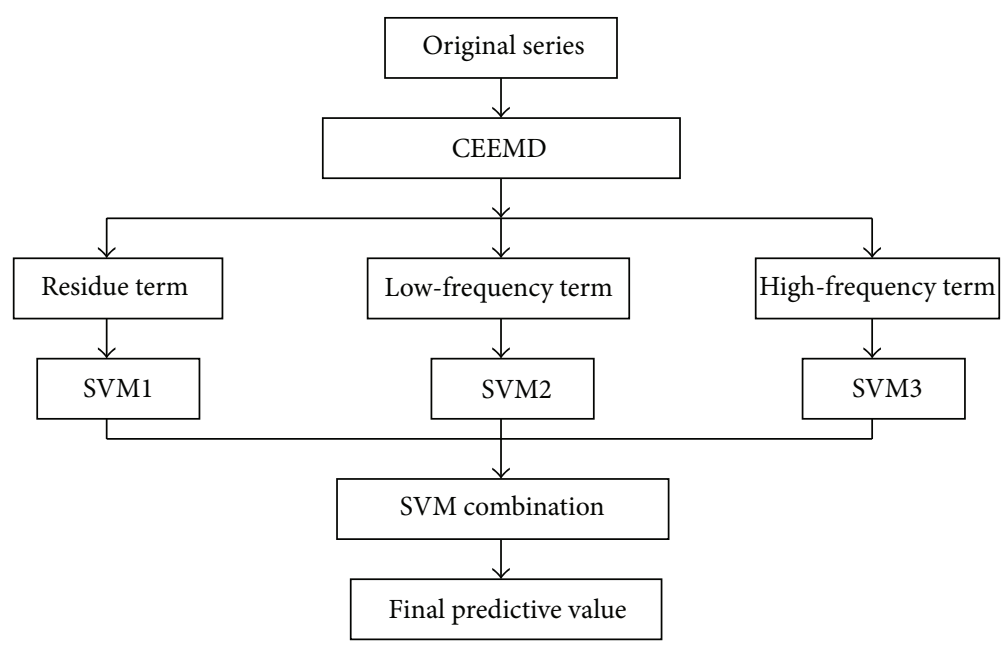

FIGURE 5: The overall process of the CEEMD-SVM-SVM model.

$$
d_{i}= \begin{cases}1, & {[x(t)-x(t-1)] *[\widehat{x}(t)-x(t-1)] \geq 0} \\ 0, & {[x(t)-x(t-1)] *[\widehat{x}(t)-x(t-1)]<0 .}\end{cases}
$$

Among the three evaluation indexes, smaller RMSE and MAPE values indicate that the prediction model is experiencing better performance, which is also true of greater DS value and better performance of the prediction model.

The prediction accuracy based on the SVM model is sensitive to the SVM model's kernel function. As many previous studies (such as $[17,22]$ ) indicate that the linear kernel function and the RBF kernel function are appropriate kernel functions for the prediction of SVM model, the linear kernel function and the RBF kernel function of the SVM are thus both applied to our prediction of Chinese stock index futures prices. Based on the process of the CEEMDSVM-SVM model, we first predict the residue term, then the low-frequency term, and then the high-frequency term of the daily price series and the five-minute price series. Notably, the five-minute price series of Chinese stock index futures is decomposed into fourteen IMFs and one residue term, where IMFs from IMF1 to IMF9 are recomposed into the high-frequency term, and IMFs from IMF10 to IMF14 are recomposed into the low-frequency term. The prediction errors of these components are shown in Table 5.

As shown in Table 5, in using the SVM model to make predictions on each component, the prediction precision of the linear kernel function SVM model is generally higher than the RBF kernel function SVM model. In addition, whether for predicting the daily price series or the fiveminute price series, the prediction precisions of the residue term and the low-frequency term are significantly higher than those of the high-frequency term. This difference occurs because these effects of shocks on the residue term and the low-frequency term are fundamental economically; they are significant events that have an impact on Chinese stock index futures prices that is sustained over a long duration.
Therefore, the fluctuations of the residue term and the lowfrequency term are relatively steady and easier to forecast using the SVM model. By contrast, the high-frequency term representing the short-term disequilibrium of Chinese stock index futures price is affected mainly by daily market activities, such as speculation, arbitrage, and the fluctuation of the high-frequency term that is random and changes rapidly. Hence, the SVM model experiences difficulty in learning the pattern of high-frequency term fluctuation, resulting in poor predictive results.

Then, according to prediction step (4), we use the optimal predictive value of each component obtained in the previous step to perform a combination prediction, and the prediction errors of our proposed model and the single SVM model based on the same data sample are simultaneously compared. These results of the prediction errors are listed in Table 6.

Table 6 shows that, as opposed to the predictive results of the three components, the RBF kernel function SVM model has higher predicted precision than the linear kernel function SVM model when performing combination prediction, which indicates that the RBF kernel function SVM model is more appropriate for combination prediction. In addition, we find that the predictive results of the final predictive value obtained under the RBF kernel function are significantly better than those obtained in the single SVM model when predicting the daily price but slightly better than the single SVM model when making predictions regarding the high-frequency price. Meanwhile, we should consider that the high-frequency price embodies far more complex information than the daily price during the same time span, which may restrict the learning ability and generalization ability of the SVM model. Hence, the CEEMD-SVM-SVM model performs better in terms of predicting the daily price. In any event, the CEEMD-SVM-SVM model proposed by this paper performs better than the single SVM model in terms of both forecasting the daily price series and the fiveminute price series. Thus, the introduction of Chinese stock index futures price characteristics in different time scales into 
TABLE 5: The prediction errors of different components.

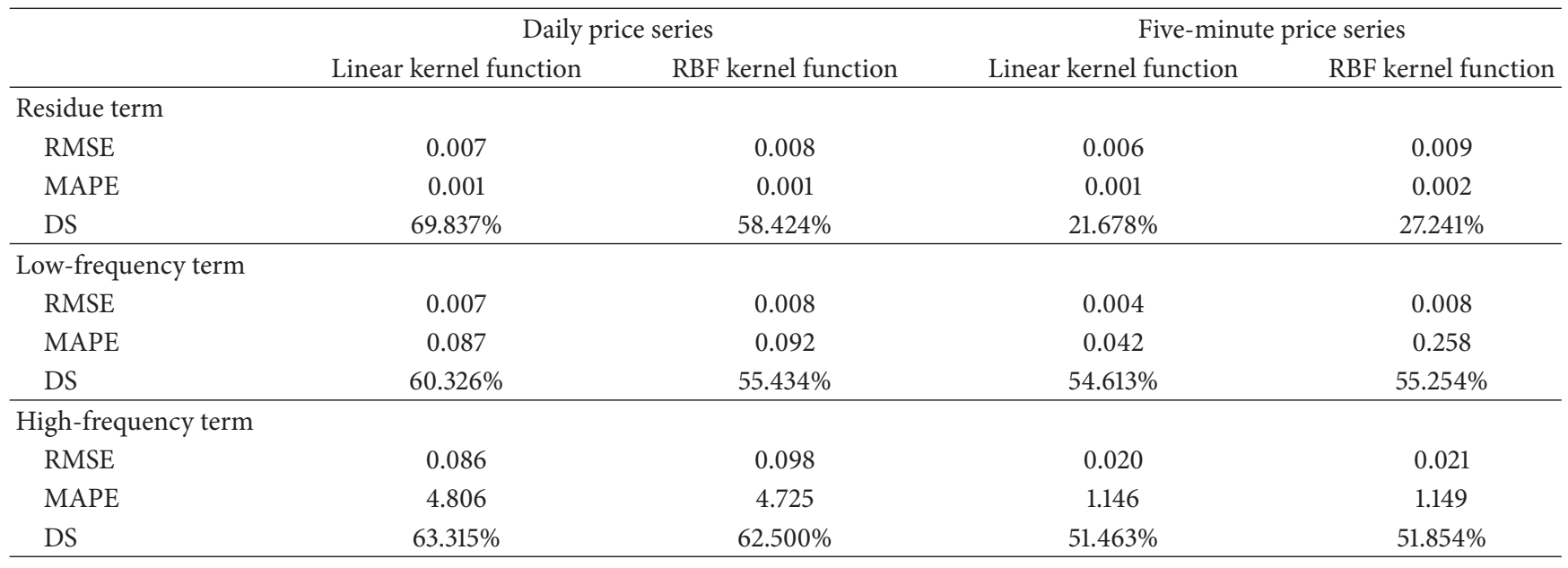

TABLE 6: Comparison of different models' prediction errors.

\begin{tabular}{lcccc}
\hline & \multicolumn{2}{c}{ Daily price series } & \multicolumn{2}{c}{ Five-minute price series } \\
& Linear kernel function & RBF kernel function & Linear kernel function & RBF kernel function \\
\hline SVM & & & & 0.008 \\
RMSE & 0.053 & 0.054 & 0.001 & 0.008 \\
MAPE & 0.009 & 0.009 & $48.171 \%$ & 0.001 \\
DS & $54.891 \%$ & $52.445 \%$ & & \\
\hline CEEMD-SVM-SVM & & & 0.008 & 0.007 \\
RMSE & 0.051 & 0.009 & 0.001 & 0.001 \\
MAPE & 0.008 & 0.002 & $51.565 \%$ & $52.715 \%$ \\
DS & $62.228 \%$ & $88.043 \%$ & & \\
\hline
\end{tabular}

the SVM model improves the ability of the SVM model to mine the variations in the stock index futures price series, further enhancing its prediction precision. Simultaneously, some useful suggestions for investors can be found in the predictions of Chinese stock index futures prices generated by our proposed model. First, investors should be accustomed to analyzing the price series from different time scales to understand the fluctuation characteristics of Chinese stock index futures prices. Second, investors must actively identify what time scale the effects of external shocks on stock index futures prices belong to and must devise the appropriate strategy accordingly. Finally, investors should pay more attention to a hedging strategy involving Chinese stock index futures over the long and/or medium term and should be cautious about short-term speculation.

Figures 6 and 7 present the real and predictive values of the daily price series and five-minute price series, respectively. The final predictive values obtained using the CEEMD-SVMSVM model appeared to coincide with the real values, indicating that the predictive values obtained using this model fit well with the real values and intuitively demonstrating that the CEEMD-SVM-SVM model is suitable for forecasting Chinese stock index futures prices.

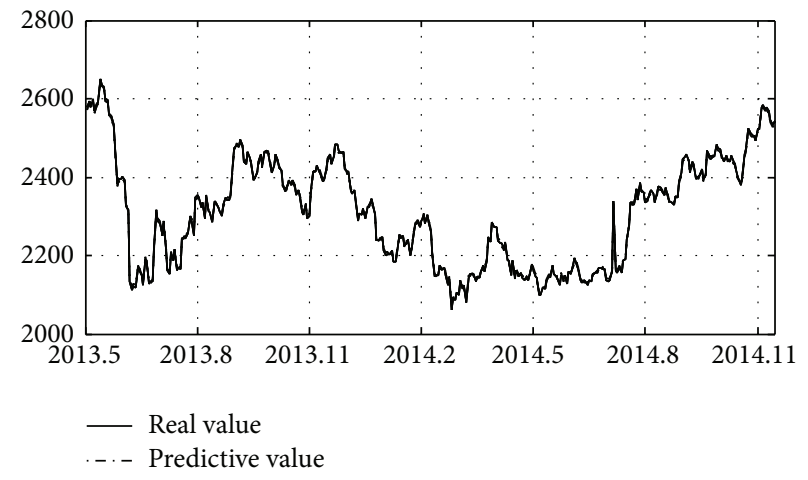

FIGURE 6: Real value and predictive value of the daily price series.

\section{Conclusion}

The Chinese stock index futures market is a complex system, and fluctuations in the stock index futures prices result from the collective effects of many factors at various levels. The CEEMD method, a method suitable in the analysis of nonlinear, nonstationary data, is applied to analyze Chinese stock index futures prices in different time scales and to 


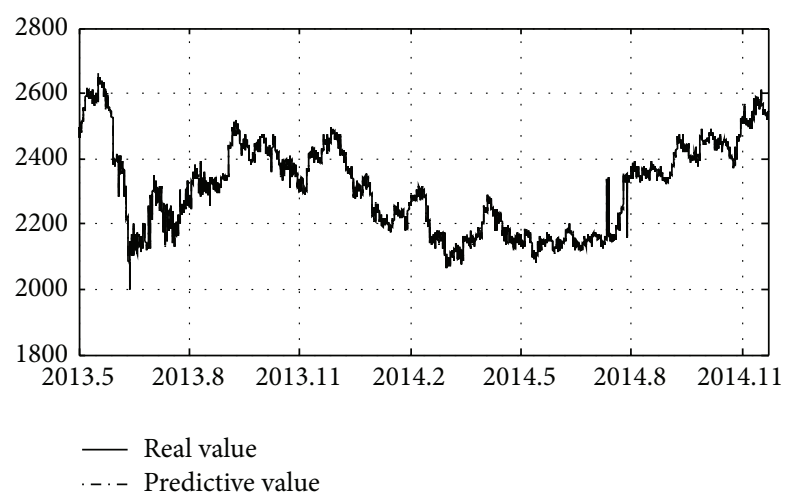

FIgURE 7: Real value and predictive value of the five-minute price series.

reveal the intrinsic fluctuation characteristics of Chinese stock index futures prices. Then, we focus on whether these characteristics improve the predictive precision of Chinese stock index futures prices and propose a CEEMD-SVM-SVM model based on the PSO algorithm. The empirical results demonstrate that the intrinsic fluctuation characteristics of Chinese stock index futures prices can be revealed in three components, including the residue term, the low-frequency term, and the high-frequency term with independent economic significance. The residue term determines the longterm trend of stock index futures prices, and its fluctuations are correlated with macroeconomics and the long-term expectations of investors. The low-frequency term reveals the effect of significant events on medium-term fluctuations in stock index futures prices; the ICSS algorithm breakpoint tests reveal that policy regulation events are the main cause of fluctuations during the low-frequency term. The highfrequency term reflects short-term disequilibrium in the stock index futures market; although the effect of the highfrequency term on the overall fluctuation in the stock index futures prices is limited, it nonetheless plays an important role locally.

In addition, in forecasting Chinese stock index futures prices, first, we find that the linear kernel function SVM model is more suitable for predicting these components than the RBF kernel function SVM model. The residue term and the low-frequency term have stronger regular fluctuations than the high-frequency term and therefore yield superior predictive results. Second, the RBF kernel function SVM model shows higher predictive precision for the final combination prediction than the linear kernel function SVM model. Simultaneously, the predictive results of the combination prediction model are better than those of a single SVM model, which indicates that the integration of fluctuation characteristics in different time scales increases the ability of the SVM model to identify regularity in Chinese stock index futures price fluctuations, thus improving prediction precision. Finally, the predictive results of the CEEMD-SVMSVM model on daily prices and intraday prices are basically consistent, which indicates that our proposed model has stability and universal applicability in forecasting Chinese stock index futures prices.

\section{Competing Interests}

The authors declare that they have no competing interests.

\section{Acknowledgments}

This work was supported in part by the National Social Science Foundation of China (14ZDA045).

\section{References}

[1] V. Meneu and H. Torró, "Asymmetric covariance in spot-futures markets," Journal of Futures Markets, vol. 23, no. 11, pp. 10191046, 2003.

[2] M. Zhong, A. F. Darrat, and R. Otero, "Price discovery and volatility spillovers in index futures markets: some evidence from Mexico," Journal of Banking and Finance, vol. 28, no. 12, pp. 3037-3054, 2004.

[3] X. Xiong, F. Wang, W. Zhang, and Y. Sun, "Price discovery and volatility spillovers between SGX FTSE/Xinhua China A50 index futures and A-share market," Chinese Journal of Management, vol. 11, no. 1535, pp. 1507-1512, 2009.

[4] H. M. Zuo, Z. T. Liu, and H. W. Zeng, "Research of volatility spillover and information transmission between stock index futures and spot market based on the high frequency data," Journal of Financial Research, vol. 4, pp. 140-154, 2012.

[5] H. Q. Chen and C. H. Zhang, "Does index futures trading reduce stock market jump risk?" Economic Research Journal, vol. 1, pp. 153-167, 2015.

[6] F. Wen, Z. He, Z. Dai, and X. Yang, "Characteristics of investors' risk preference for stock markets," Economic Computation and Economic Cybernetics Studies and Research, vol. 48, no. 3, pp. 235-254, 2015.

[7] E. E. Peters, Fractal Market Analysis: Applying Chaos Theory to Investment and Economics, John Wiley \& Sons, New York, NY, USA, 1994

[8] X. Zhang, K. K. Lai, and S.-Y. Wang, "A new approach for crude oil price analysis based on empirical mode decomposition," Energy Economics, vol. 30, no. 3, pp. 905-918, 2008.

[9] N. E. Huang, Z. Shen, S. R. Long et al., "The empirical mode decomposition and the Hilbert spectrum for nonlinear and nonstationary time series analysis," Proceedings of the Royal Society of London A, vol. 454, no. 1971, pp. 903-995, 1998.

[10] Z. Wu and N. E. Huang, "Ensemble empirical mode decomposition: a noise-assisted data analysis method," Advances in Adaptive Data Analysis, vol. 1, no. 1, pp. 1-41, 2009.

[11] L. F. Ruan and H. J. Bao, "An empirical analysis on periodic fluctuations of real estate price based on EMD," Chinese Journal of Management Science, no. 3, pp. 41-46, 2012.

[12] H. L. Li and C. E. Feng, "Relationship between investor sentiment and stock indices fluctuation based on EEMD," Systems Engineering-Theory \& Practice, vol. 10, pp. 2495-2503, 2014.

[13] J.-R. Yeh, J.-S. Shieh, and N. E. Huang, "Complementary ensemble empirical mode decomposition: a novel noise enhanced data analysis method," Advances in Adaptive Data Analysis, vol. 2, no. 2, pp. 135-156, 2010.

[14] L. Zhao, W. Yu, and R. Yan, "Rolling bearing fault diagnosis based on CEEMD and time series modeling," Mathematical Problems in Engineering, vol. 2014, Article ID 101867, 13 pages, 2014. 
[15] L. Tang, W. Dai, L. Yu, and S. Wang, "A novel CEEMDbased eelm ensemble learning paradigm for crude oil price forecasting," International Journal of Information Technology and Decision Making, vol. 14, no. 1, pp. 141-169, 2015.

[16] L. Yu, S. Wang, and K. K. Lai, "Forecasting crude oil price with an EMD-based neural network ensemble learning paradigm," Energy Economics, vol. 30, no. 5, pp. 2623-2635, 2008.

[17] Y. F. Yang, Y. K. Bao, Z. Y. Hu, and R. Zhang, "Crude oil price prediction based on empirical mode decomposition and support vector machines," Chinese Journal of Management, vol. 12, pp. 1884-1889, 2010.

[18] V. N. Vapnik, Statistical Learning Theory, John Wiley \& Sons, New York, NY, USA, 1998.

[19] W. Huang, Y. Nakamori, and S.-Y. Wang, "Forecasting stock market movement direction with support vector machine," Computers and Operations Research, vol. 32, no. 10, pp. 25132522, 2005.

[20] H. Ince and T. B. Trafalis, "Short term forecasting with support vector machines and application to stock price prediction," International Journal of General Systems, vol. 37, no. 6, pp. 677687, 2008.

[21] F. E. H. Tay and L. Cao, "Application of support vector machines in financial time series forecasting," Omega, vol. 29, no. 4, pp. 309-317, 2001.

[22] Y. Sai, F. T. Zhang, and T. Zhang, "Research of Chinese stock index futures regression prediction based on support vector machines," Chinese Journal of Management Science, vol. 3, pp. 35-39, 2013.

[23] C. Inclán and G. C. Tiao, "Use of cumulative sums of squares for retrospective detection of changes of variance," Journal of the American Statistical Association, vol. 89, no. 427, pp. 913-923, 1994.

[24] S. H. Kang, C. Cheong, and S.-M. Yoon, "Structural changes and volatility transmission in crude oil markets," Physica A: Statistical Mechanics and Its Applications, vol. 390, no. 23-24, pp. 4317-4324, 2011.

[25] J. P. Yang, X. X. Chen, and C. H. Zhang, "Policy impact factors on the volatility of Shanghai and Shenzhen stock market in China," Chinese Journal of Management Science, vol. 20, no. 6, pp. 43-51, 2012.

[26] J. Kennedy, "Particle swarm optimization," in Encyclopedia of Machine Learning, pp. 760-766, Springer, New York, NY, USA, 2010.

[27] C.-L. Huang and J.-F. Dun, "A distributed PSO-SVM hybrid system with feature selection and parameter optimization," Applied Soft Computing, vol. 8, no. 4, pp. 1381-1391, 2008.

[28] C. Zhou, K. Yin, Y. Cao, and B. Ahmed, "Application of time series analysis and PSO-SVM model in predicting the Bazimen landslide in the Three Gorges Reservoir, China," Engineering Geology, vol. 204, pp. 108-120, 2016.

[29] Z. Dai and F. Wen, "Robust CVaR-based portfolio optimization under a genal affine data perturbation uncertainty set," Journal of Computational Analysis \& Applications, vol. 16, no. 1, pp. 93103, 2014.

[30] X. Gong, Z. He, P. Li, and N. Zhu, "Forecasting return volatility of the CSI 300 index using the stochastic volatility model with continuous volatility and jumps," Discrete Dynamics in Nature and Society, vol. 2014, Article ID 964654, 10 pages, 2014.

[31] S. Q. Guo and P. H. Liang, "Social Interactions, the information channel and household stock market participation: a empirical study based on a Chinese household finance survey," Economic Research Journal, vol. S1, pp. 116-131, 2014. 


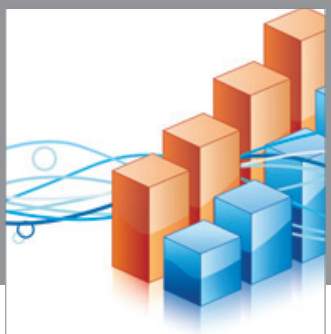

Advances in

Operations Research

vatem alat4

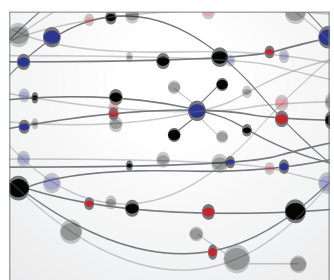

\section{The Scientific} World Journal
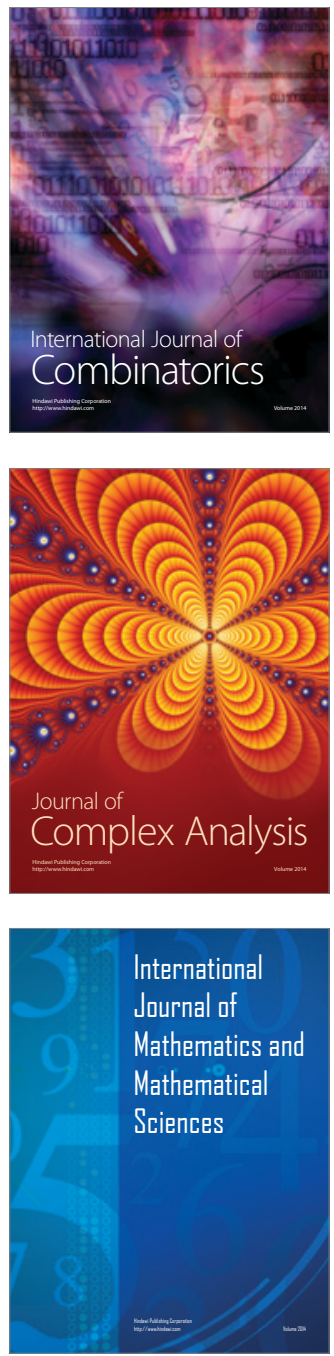
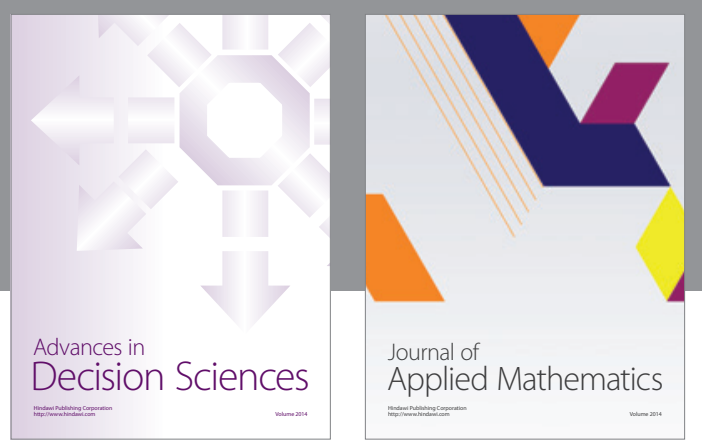

Algebra

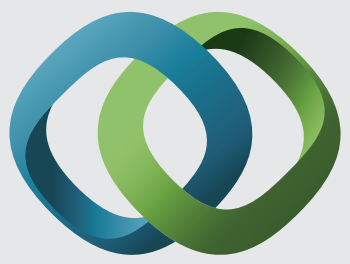

\section{Hindawi}

Submit your manuscripts at

http://www.hindawi.com
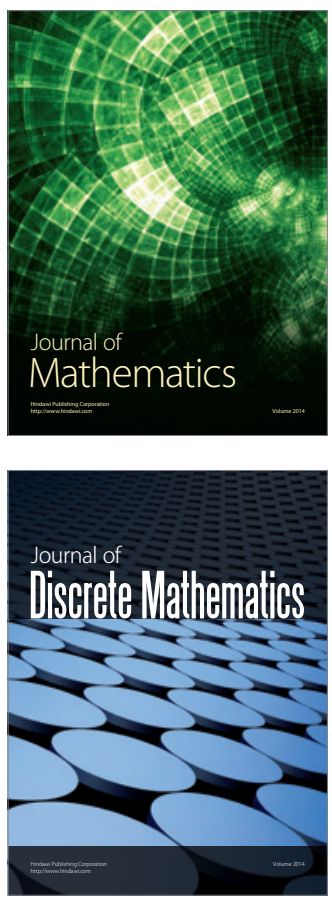

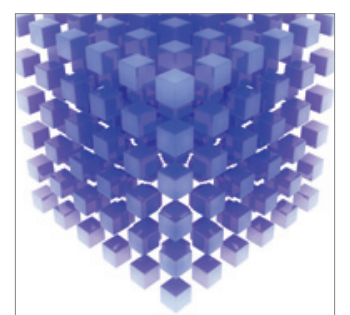

Mathematical Problems in Engineering
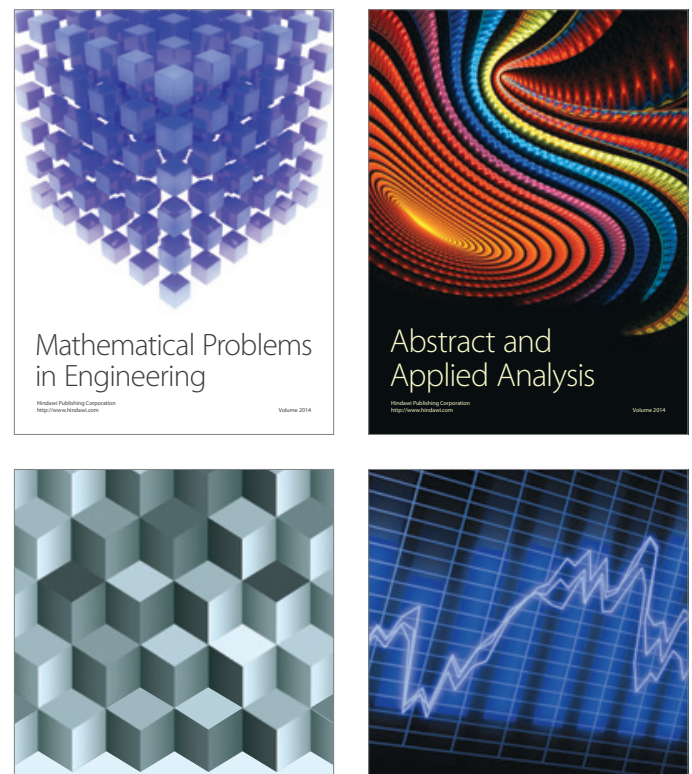

Journal of

Function Spaces

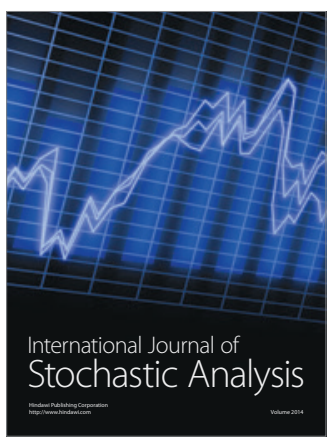

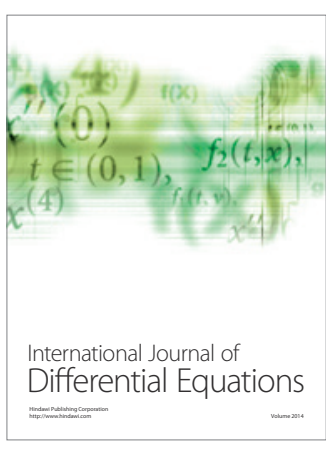
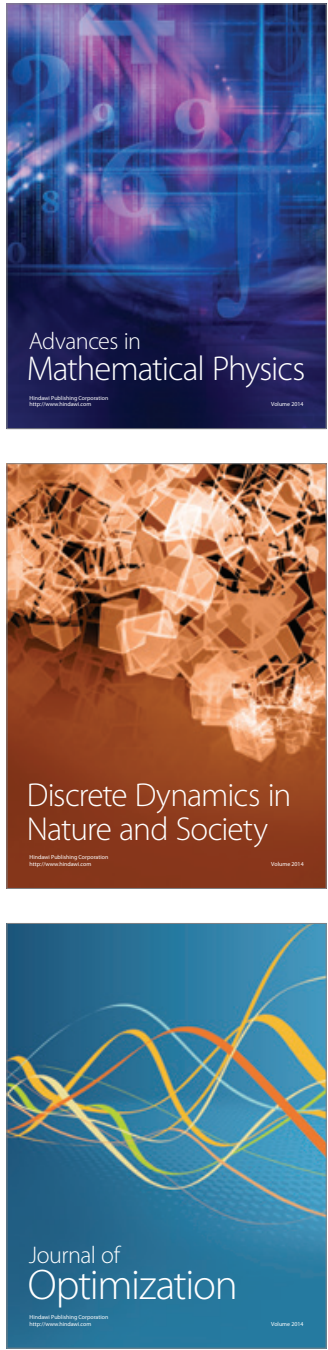